\title{
Euclidean-Space Measures of Robotic Joint Failures
}

\author{
James D. English and Anthony A. Maciejewski \\ Purdue University
1285 Electrical Engineering Building
West Lafayette, Indiana 47907-1285
}

\section{Abstract}

Robotic joint failures are directly characterized and measured in joint space. A locking failure, for example, is one for which a joint cannot move, and it gives an error equal to the desired value minus the locked value. This article extends the joint-space characterization to Euclidean space by measuring a failure's effect there. The approach is based on a primitive measure of point error that can be defined to be distance or path length. It is used to form comprehensive measures through weighted integration over Euclidean-space regions. For kinematically redundant manipulators, minimizing the measures can be used to induce failure tolerance by either reducing the likelihood of collision-induced damage before a failure or reducing end-effector error after a failure. Examples for both cases are given.

\section{INTRODUCTION}

Some examples of robotic joint-failure types are locking, where the joint cannot move [1, 2, 3]; free swinging, where actuator torque is lost [4]; and calibration, where the joint value has an offset. These failures all eventually express themselves through joint position error. Errors may involve multiple joints (as, for example, when a hydraulic system loses pressure). But it is typical-and will be assumed for this work-that a failure-induced error is isolated to one joint. This joint error is an imprecise measure of the effect of the failure, however, even for the same joint on the same manipulator, as is illustrated in Fig. 1.

This article will address the effect of failure-induced joint position error in Euclidean space. There is no one natural way to measure the kinematic aspects of rigid-body motions with a scalar [5]. However, physical objects do allow a focusing of motion to form scalar measures (an example of which is the "volume of a swept volume" [6], among others [7]), and this concept will be used here. The idea will be to first define a measure of the motion of a point after a joint failure - a simpler task - and then extend it to find the motion of an object

This work was supported by a NASA graduate student research fellowship (grant number NGT9-2) and by Sandia National Laboratories under contract number AL-3011.

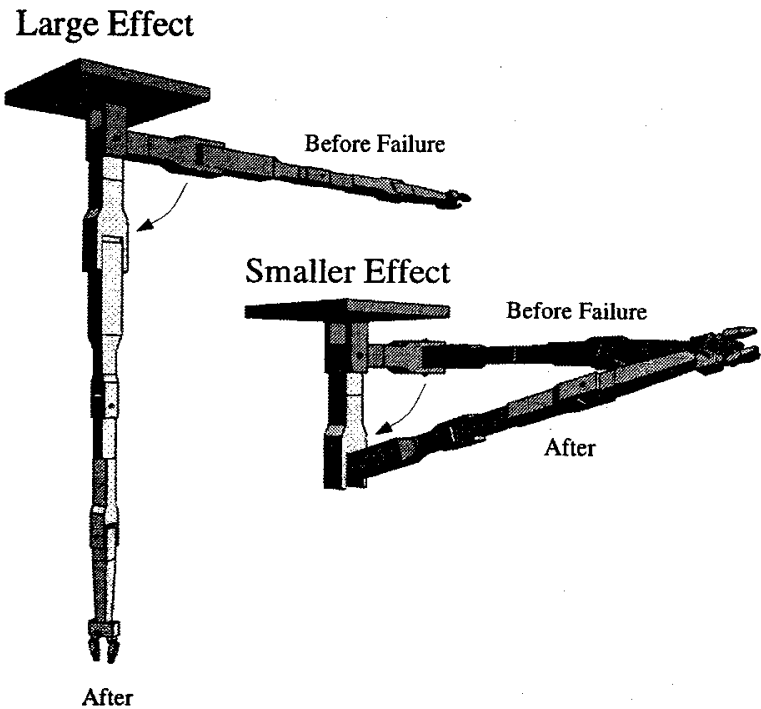

Fig. 1. A manipulator in two configurations before and after a joint-one error of $\frac{\pi}{2}$. The upper-left robot experiences extensive arm and end-effector displacement, while the lower-right robot experiences significantly less. This article will present methods to measure and reduce these kinematic effects that transcend joint error. (The error shown here would correspond to that caused by a loss of joint-one actuator torque.)

by integrating a weighting of the point measure squared over the object. The object measure will be extended to a manipulator-wide measure by incorporating multiple objects in multiple frames.

The measures will in general be functions of the joint variables, and a primary goal of this work is to enable reduction of the measures in kinematically redundant manipulators using self motion. The aim is to achieve a degree of failure tolerance by either best configuring a manipulator in anticipation of a failure or reconfiguring it for failure recovery. Failure tolerance is especially important for manipulators used in hazardous or remote environments $[8,9,10]$, and kinematically redundant manipulators have been proposed for use there $[2,11,12]$. 


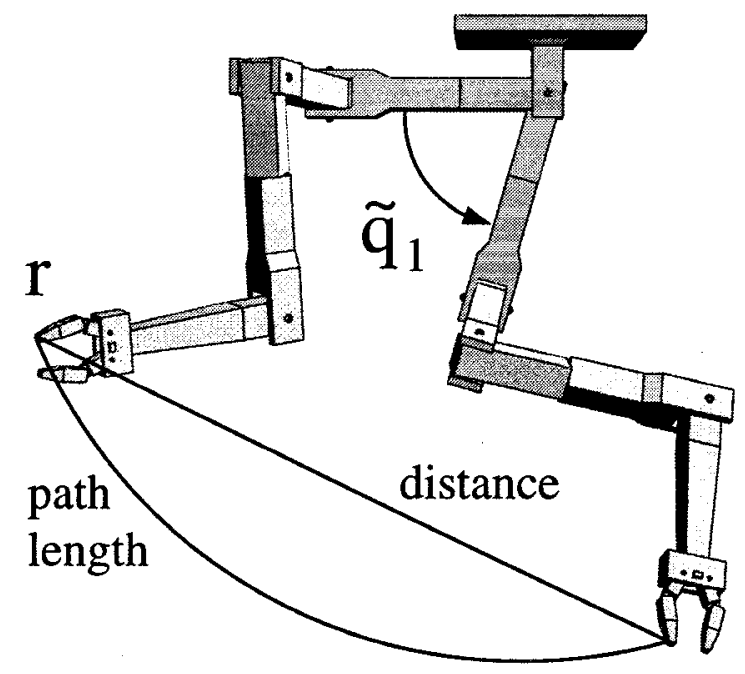

Fig. 2. A manipulator before and after a failure of the first joint. The joint error $\tilde{q}_{1}$ induces motion of point $r$ (a point on the hand in this case). Two possible measures $e_{1}$ of this motion are shown: path length assuming stationary healthy joints and Euclidean distance.

Methods for using functions to resolve redundancy include the augmented-Jacobian technique $[13,14]$ for tracking a desired value, the extended-Jacobian technique [15] for tracking critical points, and the gradientprojection method $[16,17]$ for tracking extrema. These methods all require knowledge of the function's gradient, and for this purpose, methods will be given for calculating the gradients of the measures.

\section{PoINT ERror}

The foundation of the measures will be established here by defining a primitive measure of the error of a point.

Let an $n$-degree-of-freedom manipulator with joint variables $\mathrm{q}$ have a failure at joint $i$, with an error in the failed joint variable of $\tilde{q}_{i}$ (i.e., $\tilde{q}_{i}$ equals the actual value of $q_{i}$, entry $i$ of $\mathbf{q}$, minus its desired value). The error $\tilde{q}_{i}$ may be a function of $\mathbf{q}$ (this generality allows different failure modes to be addressed). Let point $r$ lie at the tip of vector $\vec{r}$ in Denavit-Hartenberg (D-H) frame $k, 1 \leq k \leq n$. The point so chosen is completely general-any location in any frame. Then the point error $e_{i}$ will represent a measure of point $r$ 's motion caused by $\tilde{q}_{i}$. Two possible values, path length assuming stationary healthy joints and Euclidean distance are shown in Fig. 2.

Let $\vec{r}_{i-1}^{*}$ be the perpendicular vector from the line passing through $\hat{z}_{i-1}$, the $\mathrm{z}$-axis of D-H frame $i-1$, to the tip of $\vec{r}$, as shown in Fig. 3. Then in its general form, the point error is defined using a static, nonnegative function $\phi(\cdot)$ as follows:

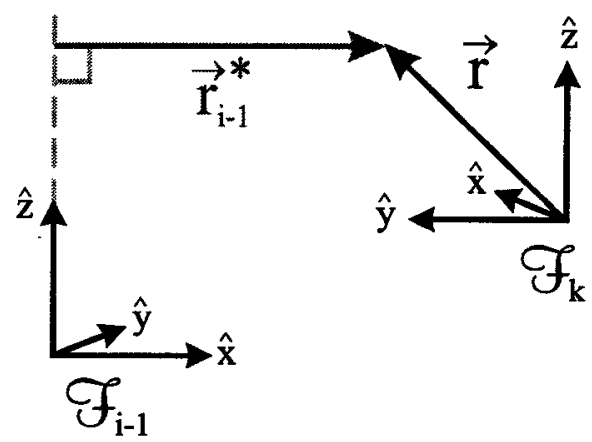

Fig. 3. To find the distance error of the tip of $\vec{r}$ caused by an error in joint $i, \vec{r}_{i-1}^{*}$ is defined as the perpendicular vector from line coinciding with the axis of joint $i$ to the tip of $\vec{r}$.

$$
\begin{aligned}
& e_{i}^{2}=\phi\left(\tilde{q}_{i}\right)\left\|\vec{r}_{i-1}^{*}\right\|^{2}, \quad i \leq k, \text { joint } i \text { rotational } \\
& \tilde{q}_{i}^{2}, \quad i \leq k, \text { joint } i \text { prismatic; } \\
& 0, \quad i>k \text {. }
\end{aligned}
$$

This general form allows $e_{i}$ to be defined as either path length assuming stationary healthy joints, using

$$
\phi\left(\tilde{q}_{i}\right)=\tilde{q}_{i}^{2},
$$

or Euclidean distance, using

$$
\phi\left(\tilde{q}_{i}\right)=2\left(1-\cos \left(\tilde{q}_{i}\right)\right) .
$$

Examples showing when each of these is applicable will be given in Section V. In the ensuing text, however, $\phi(\cdot)$ will be used in the general sense and not restricted to either of these values.

The scalar $\left\|\vec{r}_{i-1}^{*}\right\|$ can be found through

$$
\left\|\vec{r}_{i-1}^{*}\right\|=\left\|\left(\vec{p}_{i-1 \rightarrow k}+\vec{r}\right) \times \hat{z}_{i-1}\right\|,
$$

where $\vec{p}_{\ell \rightarrow k}$ is the vector from the origin of D-H frame $\ell$ to $\mathrm{D}$-H frame $k$. This equation is in coordinate-free form and can be calculated in any frame.

\section{OBject Error}

Different points on the manipulator (typically) move different distances after a failure, and a region-or object-rigidly attached to one $\mathrm{D}-\mathrm{H}$ frame will be used here to expand the primitive measure. This object may comprise several disjoint sets. The point error squared times a weighting function will be integrated over the object to find the object-based measure.

Let $\Omega_{k}$ be the object rigidly attached to frame $k$, and let $\rho_{k}(\vec{r}, t)$ be a possibly time-varying weighting function for which there exists some (preferably small) integer $N_{k}$ such that it can be decomposed as follows: 


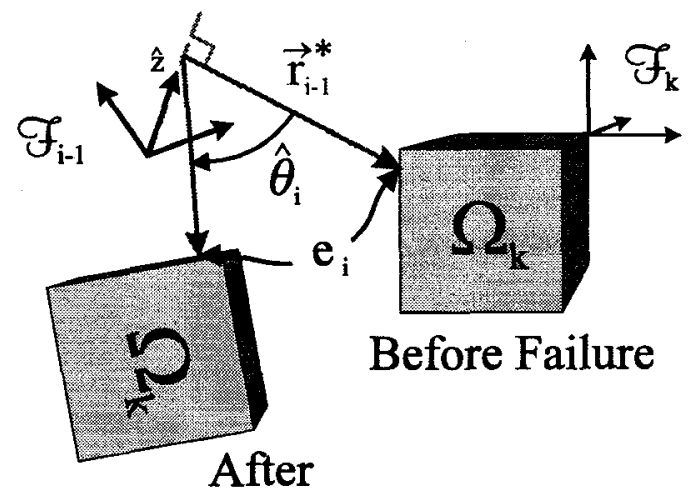

Fig. 4. To find the object error for object $\Omega_{k}$ rigidly attached to $D$-H frame $k$, the weighted point error squared is integrated over the object.

$$
\rho_{k}(\vec{r}, t)=\sum_{i=1}^{N_{k}} \rho_{k, i}^{\prime}(\vec{r}) \rho_{k, i}^{\prime \prime}(t) .
$$

This decomposition will simplify the measure's calculation.

With this, the object-based measure $o_{i}$ is established by integrating the product of $\rho_{k}(\vec{r}, t)$ and $\left[e_{i}\left(\vec{r}, \tilde{q}_{i}\right)\right]^{2}$ over $\Omega_{k}$ :

$$
o_{i}\left(\Omega_{k}, \tilde{q}_{i}, \mathbf{q}, t\right)=\int_{\Omega_{k}} \rho_{k}(\vec{r}, t)\left[e_{i}\left(\vec{r}, \tilde{q}_{i}, \mathbf{q}\right)\right]^{2} d \omega_{k},
$$

where $d \omega_{k}$ is a differential volume, area, or distance element when $\Omega_{k}$ is a solid, surface, or curve, respectively. This concept is illustrated in Fig. 4 for a solid $\Omega_{k}$. When $e_{i}$ is Euclidean distance, (6) corresponds to the object norm of Kazerounian and Rastegar [18].

Equation (6) is in general a computationally expensive calculation that cannot be performed on line. This integration has been identified as a drawback of Kazerounian and Rastegar's method [7, 19]. However, it will be shown below that for the given assumptions there exists a set of integrations independent of the joint variables that can be performed only once, and the results used in lieu of integrating $\left[\rho_{k}(\vec{r}, t) e_{i}^{2}\right]$ each time step. When $N_{k}$ is sufficiently small, this will allow realtime calculation.

\section{A. Calculating $o_{i}$ for Rotational Joint $i$}

Using (1) for joint $i$ rotational, (6) gives

$$
o_{i}=\begin{array}{ll}
\phi_{i} \int_{\Omega_{k}} \rho_{k}\left\|\vec{r}_{i-1}^{*}\right\|^{2} d \omega_{k}, & i \leq k \\
0, & i>k,
\end{array}
$$

where $\phi_{i}=\phi\left(\tilde{q}_{i}\right)$. Thus the problem becomes one of finding $\int_{\Omega_{k}} \rho_{k}\left\|\vec{r}_{i-1}^{*}\right\|^{2} d \omega_{k}$. This can be done efficiently by first calculating the following quantities off line:

$$
\begin{gathered}
{ }_{i} m_{\Omega_{k}}^{\prime}=\int_{\Omega_{k}} \rho_{k, i}^{\prime}(\vec{r}) d \omega_{k} \\
{ }_{i} \vec{h}_{\Omega_{k}}^{\prime}=\int_{\Omega_{k}} \rho_{k, i}^{\prime}(\vec{r}) \vec{r} d \omega_{k} ; \\
{ }_{i} \mathbf{I}_{\Omega_{k}}^{\prime}=\int_{\Omega_{k}} \rho_{k, i}^{\prime}(\vec{r}) \mathbf{R}^{T} \mathbf{R} d \omega_{k},
\end{gathered}
$$

where $\mathbf{R}$ is the cross-product matrix for $\vec{r}$; i.e., $\mathbf{R} \vec{v}=$ $\vec{r} \times \vec{v}$ for all $\vec{v}$. The definitions in (8), (9), and (10) are analogous to the rigid-body inertial parameters of mass, first moment of inertia, and second moment of inertia, respectively. They allow $m_{\Omega_{k}}=\int_{\Omega_{k}} \rho_{k} d \omega_{k}$, $\vec{h}_{\Omega_{k}}=\int_{\Omega_{k}} \rho_{k} \vec{r} d \omega_{k}$, and $\mathbf{I}_{\Omega_{k}}=\int_{\Omega_{k}} \rho_{k} \mathbf{R}^{T} \mathbf{R} d \omega_{k}$ to be calculated through the following on-line summations:

$$
\begin{gathered}
m_{\Omega_{k}}(t)=\sum_{i=1}^{N_{k}} \rho_{k, i}^{\prime \prime}(t)_{i} m_{\Omega_{k}}^{\prime} \\
\vec{h}_{\Omega_{k}}(t)=\sum_{i=1}^{N_{k}} \rho_{k, i}^{\prime \prime}(t)_{i} \vec{h}_{\Omega_{k}}^{\prime} \\
\mathbf{I}_{\Omega_{k}}(t)=\sum_{i=1}^{N_{k}} \rho_{k, i}^{\prime \prime}(t)_{i} \mathbf{I}_{\Omega_{k}}^{\prime}
\end{gathered}
$$

Using these values with the identity $\left\|\vec{r} \times \hat{z}_{i-1}\right\|^{2}=$ $\hat{z}_{i-1} \cdot \mathbf{R}^{T} \mathbf{R} \hat{z}_{i-1},(4)$ allows $\int_{\Omega_{k}} \rho_{k}\left\|\vec{r}_{i-1}^{*}\right\|^{2} d \omega_{k}$ to be expressed in coordinate-free form as

$$
\begin{aligned}
& \int_{\Omega_{k}} \rho_{k}\left\|\vec{r}_{i-1}^{*}\right\|^{2} d \omega_{k}=m_{\Omega_{k}}\left\|\vec{p}_{i-1 \rightarrow k} \times \hat{z}_{i-1}\right\|^{2}+ \\
& 2 \hat{z}_{i-1} \times\left(\vec{p}_{i-1 \rightarrow k} \times \hat{z}_{i-1}\right) \cdot \vec{h}_{\Omega_{k}}+\hat{z}_{i-1} \cdot \mathbf{I}_{\Omega_{k}} \hat{z}_{i-1}
\end{aligned}
$$

This used in (7) allows calculation of (6) with no on-line integration. Equation (14) can be efficiently calculated in frame $k$.

\section{B. Calculating o for Prismatic Joint $i$}

Substituting (1) for joint $i$ prismatic into (6) gives

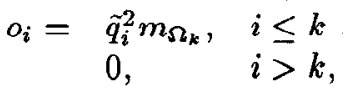

with $m_{\Omega_{k}}$ calculated using (11).

\section{Finding the Gradient}

For use in reducing $o_{i}$, its gradient will typically be used. The gradient is formed from the partial derivatives with respect to the joint variables, and methods for calculating these are presented here. 


\section{C.1 Joint $i$ Rotational}

When joint $i$ is rotational and $i>k$, from (7) $o_{i}=0$ and therefore

$$
\frac{\partial}{\partial q_{j}} o_{i}=0,
$$

and when $i \leq k$, taking the partial derivative of $(7)$ gives

$$
\begin{aligned}
\frac{\partial}{\partial q_{j}} o_{i}= & \phi_{i} \frac{\partial}{\partial q_{j}} \int_{\Omega_{k}} \rho_{k}\left\|\vec{r}_{i-1}^{*}\right\|^{2} d \omega_{k}+ \\
& {\left[\frac{\partial}{\partial q_{j}} \phi_{i}\right] \int_{\Omega_{k}} \rho_{k}\left\|\vec{r}_{i-1}^{*}\right\|^{2} d \omega_{k} . }
\end{aligned}
$$

In finding $\frac{\partial}{\partial q_{j}} \int_{\Omega_{k}} \rho_{k}(\vec{r})\left\|\vec{r}_{i-1}^{*}\right\|^{2} d \omega_{k}$, for $i \geq j$ or $k<j$, $\left\|\vec{r}_{i-1}^{*}\right\|$ does not change as a function of $q_{j}$ and thus

$$
\frac{\partial}{\partial q_{j}} \int_{\Omega_{k}} \rho_{k}(\vec{r})\left\|\vec{r}_{i-1}^{*}\right\|^{2} d \omega_{k}=0
$$

This leaves only the case $i<j \leq k$. When joint $j$ is rotational, applying the product rule to (14) and using the fact that outboard, fixed-length vectors move according to the rule $\frac{\partial}{\partial q_{j}} \vec{v}=\hat{z}_{j-1} \times \vec{v}$, gives

$$
\begin{aligned}
& \frac{\partial}{\partial q_{j}} \int_{\Omega_{k}} \rho_{k}(\vec{r})\left\|\vec{r}_{i-1}^{*}\right\|^{2} d \omega_{k}= \\
& 2\left(\left(\left(m_{\Omega_{k}} \vec{p}_{i-1 \rightarrow k}+\vec{h}_{\Omega_{k}}\right) \times \hat{z}_{i-1}\right) \cdot\left(\left(\hat{z}_{j-1} \times \vec{p}_{j-1 \rightarrow k}\right) \times \hat{z}_{i-1}\right)+\right. \\
& \left.\hat{z}_{i-1} \times\left(\vec{p}_{i-1 \rightarrow k} \times \hat{z}_{i-1}\right) \cdot \hat{z}_{j-1} \times \vec{h}_{\Omega_{k}}-\hat{z}_{j-1} \times \hat{z}_{i-1} \cdot \mathbf{I}_{\Omega_{k}} \hat{z}_{i-1}\right) .
\end{aligned}
$$

When joint $j$ is prismatic, only $\vec{p}_{i-1 \rightarrow k}$ changes with $q_{j}$, according to the rule $\frac{\partial}{\partial q_{j}} \vec{p}_{i-1 \rightarrow k}=\hat{z}_{j-1}$, giving

$$
\begin{aligned}
& \frac{\partial}{\partial q_{j}} \int_{\Omega_{k}} \rho_{k}(\vec{r})\left\|\vec{r}_{i-1}^{*}\right\|^{2} d \omega_{k}= \\
& 2\left(\hat{z}_{j-1} \times \hat{z}_{i-1}\right) \cdot\left(\left(m_{\Omega_{k}} \vec{p}_{i-1 \rightarrow k}+\vec{h}_{\Omega_{k}}\right) \times \hat{z}_{i-1}\right) .
\end{aligned}
$$

\section{C.2 Joint $i$ Prismatic}

When joint $i$ is prismatic, from (15),

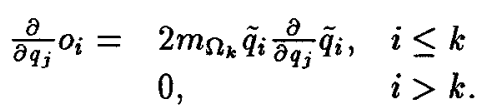

\section{Multi-Object Error}

Section III presented a measure of joint error that assigned a scalar to the movement of an object rigidly attached to one frame. This was, however, restrictive. If secondary damage caused by a moving manipulator after a failure is of concern, for example, then the entire arm should be taken into account. This section presents a measure for this purpose and gives an efficient calculation method. The measure is established through a weighted integration of the point error squared over multiple objects in multiple frames.
For all $k, 1 \leq k \leq n$, let $\Omega_{k}$ be the object rigidly attached to $\mathrm{D}$-H frame $k$, with $\rho_{k}(\vec{r}, t)$ the weighting function for $\Omega_{k}$. Then integrating the weighting of $e_{i}^{2}$ over all the regions is equivalent to summing $n$ singleobject-based measures. Using $o_{i}=0$ for $i>k$, it becomes

$$
\hat{o}_{i}=\sum_{k=i}^{n} o_{i}\left(\Omega_{k}\right) \text {. }
$$

With this formulation, $\hat{o}_{i}$ could be found by repeated application of (7) and (15). However, this can be an inefficient approach if $\hat{o}_{i}$ is calculated for multiple values of $i$. A procedure is given below that allows calculation of $\hat{o}_{i}$ for all $i, 1 \leq i \leq n$, in order $\mathrm{O}(n)$ time once the $\tilde{q}_{i}$ are known.

\section{A. Calculating $\hat{o}_{i}$ for Rotational Joint $i$}

Using (7) in (22) and factoring out $\phi_{i}$ gives

$$
\hat{o}_{i}=\phi_{i} \sum_{k=i}^{n} \int_{\Omega_{k}} \rho_{k}\left\|\vec{r}_{i-1}^{*}\right\|^{2} d \omega_{k}
$$

Now, if $\mathbf{I}_{\Omega_{k}}^{\ell}$ is defined as

$\mathbf{I}_{\Omega_{k}}^{\ell}=m_{\Omega_{k}} \mathbf{P}_{\ell \rightarrow k}^{T} \mathbf{P}_{\ell \rightarrow k}+\mathbf{P}_{\ell \rightarrow k}^{T} \mathbf{H}_{\Omega_{k}}+\mathbf{H}_{\Omega_{k}}^{T} \mathbf{P}_{\ell \rightarrow k}+\mathbf{I}_{\Omega_{k}}$,

where $\mathbf{P}_{\ell \rightarrow k}$ is the cross-product matrix for $\vec{p}_{\ell \rightarrow k}$ and $\mathbf{H}_{\Omega_{k}}$ is the cross-product matrix for $\vec{h}_{\Omega_{k}}$, and $\mathbf{I}_{\ell}^{*}$ is defined as

$$
\mathbf{I}_{\ell}^{*}=\sum_{k=\ell+1}^{n} \mathbf{I}_{\Omega_{k}}^{\ell},
$$

then, using (14), (23) becomes

$$
\hat{o}_{i}=\phi_{i} \hat{z}_{i-1} \cdot \mathbf{I}_{i-1}^{*} \hat{z}_{i-1} \text {. }
$$

The matrix $\mathbf{I}_{\ell}^{*}$ as defined through (24) and (25) is analogous to the second moment of composite rigidbody inertia [20]; it can be calculated through the following procedure: Let the net outboard interest be $\mho_{\ell}$, calculated using

$$
\mho_{\ell}=m_{\Omega_{\ell}}+\mho_{\ell+1} ; \mho_{n}=m_{\Omega_{n}} .
$$

And let $\vec{h}_{\ell}^{*}$ be the outboard interest vector, calculated using

$$
\vec{h}_{\ell}^{*}={ }^{\ell} \mathbf{R}_{\ell+1}\left(\vec{h}_{\ell+1}^{*}+\vec{h}_{\Omega_{\ell+1}}+\mho_{\ell+1} \vec{p}_{\ell+1}\right) ; \vec{h}_{n}^{*}=\overrightarrow{0},
$$

where for calculation $\vec{h}_{\Omega_{\ell+1}}$ and $\vec{p}_{\ell+1}$ are both expressed in $\mathrm{D}-\mathrm{H}$ frame $\ell+1$. Then $\mathbf{I}_{\ell}^{*}$ can be found through the following recursion: 


$$
\begin{gathered}
\mathbf{I}_{\ell}^{*}={ }^{\ell} \mathbf{R}_{\ell+1}\left(\mathbf{I}_{\ell+1}^{*}+\mathbf{I}_{\Omega_{\ell+1}}-\mathbf{P}_{\ell \rightarrow \ell+1}\left(\mathbf{H}_{\ell+1}^{*}+\mathbf{H}_{\Omega_{\ell+1}}\right)-\right. \\
\left.\left(\mathbf{H}_{\ell+1}^{*}+\mathbf{H}_{\Omega_{\ell+1}}\right) \mathbf{P}_{\ell \rightarrow \ell+1}-\mho_{\ell+1} \mathbf{P}_{\ell \rightarrow \ell+1} \mathbf{P}_{\ell \rightarrow \ell+1}\right)^{\ell} \mathbf{R}_{\ell+1}^{T} \\
\mathbf{I}_{n}^{*}=\mathbf{0} .
\end{gathered}
$$

Each of (27), (28), and (29) with (30) can be calculated in $\mathrm{O}(n)$ time.

\section{B. Calculating $\hat{o}_{i}$ for Prismatic Joint $i$}

When joint $i$ is prismatic, $\hat{o}_{i}$ is given by

$$
\hat{o}_{i}=\tilde{q}_{i}^{2} \mho_{i}
$$

with $\mho_{i}$ calculated using $(27)$.

\section{Finding the Gradient}

The technique used to find the value of $\hat{o}_{i}$ in $O(n)$ time will be used here to establish a method for finding the gradients. It will enable calculation of $\nabla \hat{o}_{i}$ for all $i$ in $O\left(n^{2}\right)$ time once the values for $\tilde{q}_{i}$ and $\nabla \tilde{q}_{i}$ are known.

\section{C.1 Joint $i$ Rotational}

From (26),

$$
\frac{\partial}{\partial q_{j}} \hat{o}_{i}=\phi_{i} \hat{z}_{i-1} \cdot\left[\frac{\partial}{\partial q_{j}} \mathbf{I}_{i-1}^{*}\right] \hat{z}_{i-1}+\left[\frac{\partial}{\partial q_{j}} \phi_{i}\right] \hat{z}_{i-1} \cdot \mathbf{I}_{i-1}^{*} \hat{z}_{i-1} \text {, }
$$

for $\frac{\partial}{\partial q_{j}} \mathbf{I}_{i-1}^{*}$ evaluated in frame $i$. When $j \leq i$, the lower right-hand element of $\mathbf{I}_{i-1}^{*}$ does not change in its own frame as $q_{j}$ changes, and (32) gives

$$
\frac{\partial}{\partial q_{j}} \hat{o}_{i}=\left[\frac{\partial}{\partial q_{j}} \phi_{i}\right] \hat{z}_{i-1} \cdot \mathbf{I}_{i-1}^{*} \hat{z}_{i-1} \text {. }
$$

When $j>i$ and joint $j$ is rotational, $\frac{\partial}{\partial q_{j}} \mathbf{I}_{i-1}^{*}$ can be found using the analogy with composite rigid-body inertia (a formula for partial derivatives is given in [4]):

$$
\begin{aligned}
& \frac{\partial}{\partial q_{j}} \hat{o}_{i}=2 \phi_{i}\left(\hat{z}_{i-1} \times \hat{z}_{j-1} \cdot \mathbf{I}_{j-1}^{*} \hat{z}_{i-1}+\right. \\
& \left(\hat{z}_{i-1} \times \vec{p}_{i-1 \rightarrow j-1}\right) \cdot\left(\vec{h}_{j-1}^{*} \times\left(\hat{z}_{j-1} \times \hat{z}_{i-1}\right)-\right. \\
& \left.\left.\hat{z}_{j-1} \times\left(\vec{h}_{j-1}^{*} \times \hat{z}_{i-1}\right)\right)\right)+\left[\frac{\partial}{\partial q_{j}} \phi_{i}\right] \hat{z}_{i-1} \cdot \mathbf{I}_{i-1}^{*} \hat{z}_{i-1}
\end{aligned}
$$

Similarly, when $j>i$ and joint $j$ is prismatic,

$$
\begin{aligned}
\frac{\partial}{\partial q_{j}} \hat{o}_{i}= & 2 \mho_{j} \phi_{i}\left(\vec{p}_{i-1-j-1} \times \hat{z}_{i-1}\right) \cdot\left(\hat{z}_{j-1} \times \hat{z}_{i-1}\right)+ \\
& {\left[\frac{\partial}{\partial q_{j}} \phi_{i}\right] \hat{z}_{i-1} \cdot \mathbf{I}_{i-1}^{*} \hat{z}_{i-1} . }
\end{aligned}
$$

\section{C.2 Joint $i$ Prismatic}

Independent of joint $j$ type, when joint $i$ is prismatic, from (31)

$$
\frac{\partial}{\partial q_{j}} \hat{o}_{i}=2 \mho_{i} \tilde{q}_{i} \frac{\partial}{\partial q_{j}} \tilde{q}_{i}
$$

\section{EXAMPLES}

In this section, the Robotics Research Corporation K-1207i manipulator will be used as an example arm. Its D-H parameters (based on the labeling scheme of Paul [21]) are given in Table $I$, and the software joint limits are given in Table II. This seven-degreeof-freedom arm has one degree of redundancy for the task of hand positioning and orienting. It is this extra degree of freedom that will be used in the examples to reduce the error measures.

\begin{tabular}{||c||r|r|r|c||}
\hline link & $a(\mathrm{~m})$ & $d(\mathrm{~m})$ & $\alpha(\mathrm{rad})$ & $\theta(\mathrm{rad})$ \\
\hline 1 & -0.1016 & 0.0000 & -1.5708 & $q_{1}$ \\
\hline 2 & 0.1016 & 0.0000 & 1.5708 & $q_{2}$ \\
\hline 3 & -0.0857 & 0.5461 & -1.5708 & $q_{3}$ \\
\hline 4 & 0.0857 & 0.0000 & 1.5708 & $q_{4}$ \\
\hline 5 & -0.0591 & 0.5461 & -1.5708 & $q_{5}$ \\
\hline 6 & 0.0591 & 0.0000 & 1.5708 & $q_{6}$ \\
\hline 7 & 0.0000 & 0.1778 & 0.0000 & $q_{7}$ \\
\hline
\end{tabular}

TABLE I

D-H parameters for the RRC K-1207i.

\begin{tabular}{||c||r|r||}
\hline joint & upper limit & lower limit \\
\hline 1 & 3.1410 & -3.1410 \\
\hline 2 & -0.0543 & -3.0510 \\
\hline 3 & 0.0000 & -6.2800 \\
\hline 4 & 0.0000 & -3.0510 \\
\hline 5 & 6.2800 & -6.2800 \\
\hline 6 & 0.6100 & -2.9670 \\
\hline 7 & 6.2800 & -6.2800 \\
\hline
\end{tabular}

TABLE II

Joint limits in radians for the RRC K-1207i.

\section{A. A Single-Object Example}

In this section, an object rigidly attached to the K-1207i's end effector will be used as $\Omega_{n}$ to measure the effect of a calibration error. To focus on a calibration error, $\tilde{q}_{i}$ will be fixed at a nonzero value. The object will be $\mathrm{L}$-shaped, formed by joining four cubes of edge length $0.06 \mathrm{~m}$. The top of the L-shaped object will lie $0.10 \mathrm{~m}$ from the end frame along the z-axis. 
For this object with $\rho_{n}=1 \mathrm{~m}^{-3}$ and Euclidean distance used as a primitive (i.e., $\phi(\cdot)$ is given by (3)), the hand was constrained to a pose given by the following homogeneous transformation matrix relative to the base frame:

$$
\mathbf{H}=\left[\begin{array}{cccc}
0 & 0 & -1 & -0.25 \\
0 & 1 & 0 & -0.60 \\
1 & 0 & 0 & 0 \\
0 & 0 & 0 & 1
\end{array}\right]
$$

With this, the gradient projection technique was used to find the worst-case and best-case configurations for tolerating a joint-five failure. These are shown in Fig. 5.

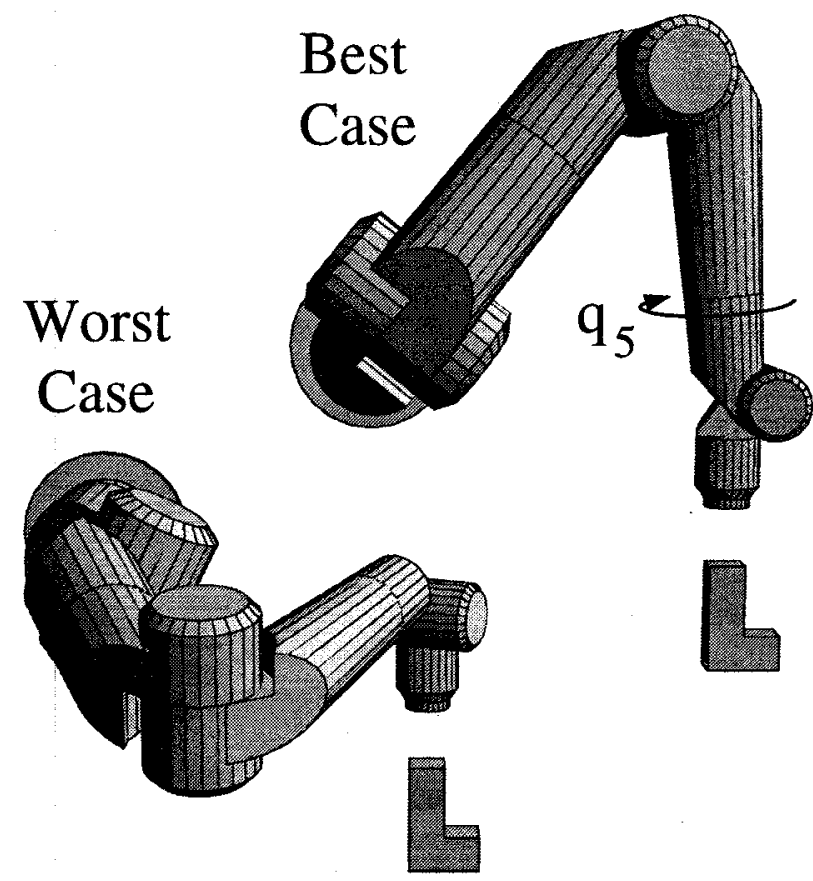

Fig. 5. Worst-case and best-case configurations of the example manipulator for reducing the effect of joint-five error on the L-shaped object under the constraint of end-effector position/orientation given by (37). In the worst case, the line passing through the axis of the fifth joint lies far from all points on the object, and in the best case, the line passing through the axis of the fifth joint actually passes through the object. The motion of the object for these two configurations after a 0.1 radian error is shown at the bottom of Fig, 6 .

The potential error in the L-shaped object caused by an error in joint five is greatly reduced by reconfiguring. In fact, $o_{5}$ for the best-case configuration of Fig. 5 is two orders of magnitude less than that of the worst case. The errors in the object for these configurations caused by a 0.1 radian error in joint five are shown along with the errors for joints two through four in Fig. 6. The improvement for a focus on joints two and five is especially substantial, but improvement is evident in all cases. If calibration had been lost in one

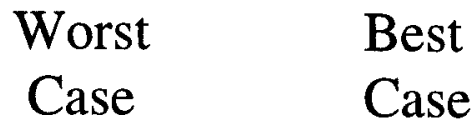

Joint Two

Ratio: 326
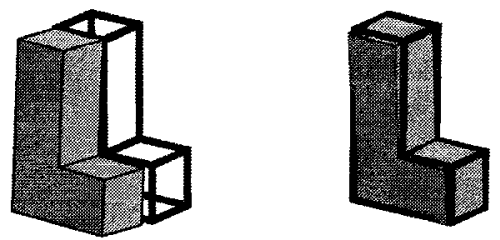

Joint Three

Ratio: 4
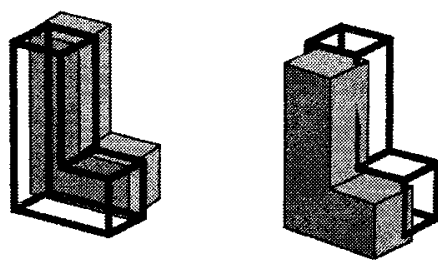

Joint Four

Ratio: 14
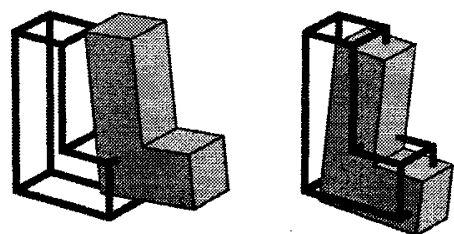

\section{Joint Five \\ Ratio: 159}
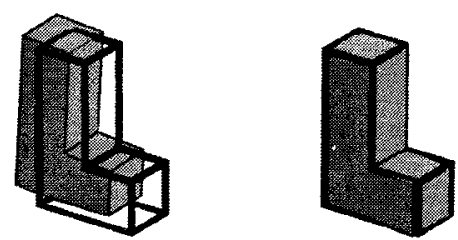

Fig. 6. Under the constraint of hand pose given by (37), the arm was placed in worst-case and best-case configurations for each of joints two through five. (The configurations for joint five are shown in Fig. 5.) Then an error of 0.1 radians was imposed on the focus joint. The resulting error in the L-shaped object is shown here. In each case, the black frame represents where the object would be if there were no error. Given to the left of each set is the ratio of the worst-case to best-case values of $o_{i}$. Some jointfailure effects are more amenable to reconfiguration than othersfor joint two the error is reduced by a factor of 326 , while for joint three the error is reduced by only a factor of four.

of these joints, reconfiguring might allow a task to be completed that would otherwise be impossible.

The cases for joints one and seven are not shown in Fig. 6 because, for static $\tilde{q}_{i}, o_{1}$ and $o_{n}$ are static once the hand is fixed. This will be the case for any manipulator where the focus is on a single object in the last frame. The case for joint six is not shown because $o_{6}$ here changes very little with reconfiguring.

This technique is also useful for addressing other failure modes in manipulators with software or hardware error checking. Error checking is commonly used in robotic controllers to stop the arm when a joint deviates excessively from its expected value, and the techniques presented in this article allow enhancement of this safety feature. If the excessive-error cutoff value 
for the K-1207i were set to 0.1 radians, it is clear from Fig. 6 that the L-shaped object would be less exposed to collision-induced damage if the manipulator were optimally configured.

\section{B. A Multi-Object, Free-Swinging-Failure Example}

In the previous example, the joint error was assumed fixed. This is not always the case, however, and for this section's example, the joint failure is of the freeswinging type, i.e., one where actuator torque is lost. After a free-swinging failure, the arm moves under the influence of gravity. If the failed joint does not hit a stop, it settles into a configuration with the center of mass of the outboard links at its lowest point relative to the gravitational field [4], and this motion is a function of configuration.

For this example, the point error is path length assuming stationary healthy joints, with $\phi(\cdot)$ given by (2). This choice is appropriate for reducing the likelihood of collision-induced damage after a failure, and the stationary-healthy-joint assumption is an approximation for a slow-moving manipulator. (Manipulators used in remote and hazardous environments are typically slow moving.) Additionally for this example, the objects $\Omega_{k}$ are the CAD models of the K-1207i links used to make the images of the robot for this article, and the weighting function is $\rho=1 \mathrm{~m}^{-3}$. The values for the free-swinging joint error and its partial derivatives are calculated using the techniques given in [4].

For the hand pose given by (37) and link masses and centers of mass given in Table III, the best-case and worst-case configurations for tolerating a free-swinging failure of the first joint are shown in Fig. 7, both before and after a failure. With $\hat{o}_{1}$ equal to 0.038 , the motion for the best-case configuration is kinematically equivalent to translating a $1 \mathrm{~m}$ cube by $19 \mathrm{~cm}$. In contrast, with $\hat{o}_{1}$ equal to 0.384 , the motion for the worst-case configuration is kinematically equivalent to translating a $1 \mathrm{~m}$ cube by $62 \mathrm{~cm}$. It is clear from Fig. 7 that this reduced motion corresponds to a reduced likelihood of collision with the environment.

\section{SUmmarY}

In this article, measures of joint failures were defined using Euclidean-space objects rigidly attached to a manipulator's links. The objects were used to expand point-error-based measures by integrating a weighting of the measures squared over the objects. Efficient ways of calculating the measures were given, and for the purpose of instilling fault tolerance in redundant manipulators, ways to calculate the measures' gradients were also presented. Examples showed how the measures could be

\begin{tabular}{||c||r|r|r|r||}
\hline link & mass $(\mathrm{kg})$ & $c_{x}(\mathrm{~m})$ & $c_{y}(\mathrm{~m})$ & $c_{z}(\mathrm{~m})$ \\
\hline 1 & 19.051 & 0 & 0 & -0.0030 \\
\hline 2 & 9.299 & 0 & 0 & 0.3239 \\
\hline 3 & 11.113 & 0 & 0 & 0.0064 \\
\hline 4 & 5.897 & 0 & 0 & 0.3200 \\
\hline 5 & 4.536 & 0 & 0 & 0.0127 \\
\hline 6 & 2.381 & 0 & 0 & 0.1219 \\
\hline 7 & 0.325 & 0 & 0 & -0.0200 \\
\hline
\end{tabular}

TABLE III

Masses and centers of mass for the RRC K-1207i.

used to prepare for a failure by reducing the likelihood of a collision or compensate for a failure by reducing the task error. Many of the reductions in collision likelihood and task error were substantial.

\section{REFERENCES}

[1] A. A. Maciejewski, "Fault tolerant properties of kinematically redundant manipulators," in Proc. 1990 IEEE International Conference on Robotics and Automation, pp. 638642, (Cincinnati, OH), May 13-18 1990.

[2] C. J. J. Paredis, W. K. F. Au, and P. K. Khosla, "Kinematic design of fault tolerant manipulators," Computers and Electrical Engineering, vol. 20, no. 3, pp. 211-220, May 1994.

[3] R. G. Roberts and A. A. Maciejewski, "A local measure of fault tolerance for kinematically redundant manipulators," IEEE Transactions on Robotics and Automation, vol. 12, no. 4, pp. 543-552, August 1996.

[4] J. D. English and A. A. Maciejewski, "Fault tolerance for kinematically redundant manipulators: Anticipating freeswinging joint failures," in Proc. 1996 IEEE International Conference on Robotics and Automation, pp. 460-467, (Minneapolis, MN), April 22-28 1996.

[5] F. C. Park and R. W. Brockett, "Kinematic dexterity of robotic mechanisms," International Journal of Robotics Research, vol. 13, no. 1, pp. 1-15, February 1994.

[6] D. Blackmore and M. Leu, "Analysis of swept volume via Lie groups and differential equations," International Journal of Robotics Research, vol. 11, no. 6, pp. 516-537, December 1992.

[7] J. M. R. Martinez and J. Duffy, "On the metrics of rigid body displacements for infinite and finite bodies," $A S M E$ Journal of Mechanical Design, vol. 117, pp. 41-47, March 1995.

[8] R. Colbaugh and M. Jamshidi, "Robot manipulator control for hazardous waste-handling applications," Journal of Robotic Systems, vol. 9, no. 2, pp. 215-250, 1992.

[9] E. Wu, J. Hwang, and J. Chladek, "Fault tolerant joint development for the space shuttle remote manipulator system:Analysis and experiment," in Proc. Fourth International Symposium on Robotics and Manufacturing (ISRAM '92), pp. 505-510, (Sante Fe, New Mexico), November 11-13 1992.

[10] M. L. Visinsky, J. R. Cavallaro, and I. D. Walker, "A dynamic fault tolerance framework for remote robots," IEEE Transactions on Robotics and Automation, vol. 11, no. 4, pp. 477-490, August 1995. 
[11] C. L. Lewis and A. A. Maciejewski, "Dexterity optimization of kinematically redundant manipulators in the presence of failures," Computers and Electrical Engineering, vol. 20, no. 3, pp. 273-288, May 1994.

[12] Y. Ting, S. Tosunoglu, and D. Tesar, "A control structure for fault-tolerant operation of robotic manipulators," in Proc. 1993 IEEE International Conference on Robotics and Automation, pp. 684-690, (Atlanta, Georgia), May 2-6 1993.

[13] O. Egeland, "Task-space tracking with redundant manipulators," IEEE Journal of Robotics and Automation, vol. RA-3, no. 5, pp. 471-475, October 1987.

[14] H. Seraji, "Configuration control of redundant manipulators: Theory and implementation," IEEE Transactions on Robotics and Automation, vol. 5, no. 4, pp. 472-490, August 1989.

[15] J. Baillieul, "Kinematic programming alternatives for redundant manipulators," in Proc. 1985 IEEE International Conference on Robotics and Automation, pp. 722-728, (St. Louis, MO), March 25-28 1985.

[16] A. Liégeois, "Automatic supervisory control of the configuration and behavior of multibody mechanisms," IEEE Transactions on Systems, Man, and Cybernetics, vol. SMC-7, no. 12, pp. 868-871, December 1977.

[17] C. A. Klein and C. H. Huang, "Review of pseudoinverse control for use with kinematically redundant manipulators," IEEE Transactions on Systems, Man, and Cybernetics, vol. SMC-13, no. 2, pp. 245-250, March/April 1983.

[18] K. Kazerounian and J. Rastegar, "Object norms: a class of coordinate and metric independent norms for displacements," in Proc. Flexible Mechanisms, Dynamics, and Analysis, pp. 271-275, (Scottsdale, Arizona), September 13-16 1992.

[19] F. C. Park, "Distance metrics on the rigid-body motions with applications to mechanical design," ASME Journal of Mechanical Design, vol. 117, pp. 48-54, March 1995.

[20] M. W. Walker and D. E. Orin, "Efficient dynamic computer simulation of robotic mechanisms," ASME Journal of Dynamic Systems, Measurement, and Control, vol. 104, pp. 205-211, September 1982.

[21] R. P. Paul, Robot Manipulators: Mathematics, Programming, and Control, MIT Press, Cambridge, Mass., 1981.

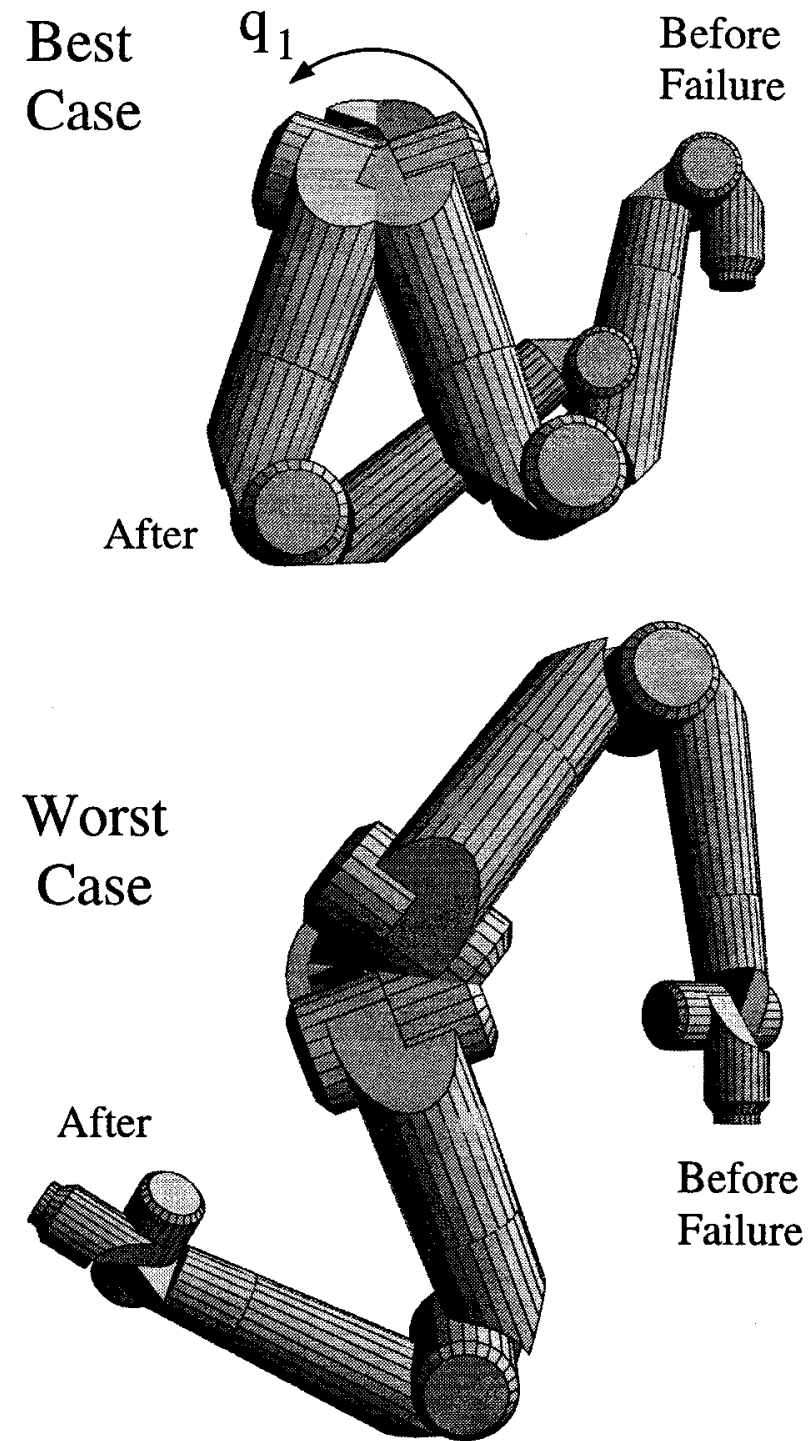

Fig. 7. Under the constraint of hand pose given by (37), the arm was placed in worst-case and best-case configurations for a free-swinging failure of the first joint. Configurations before and after a failure are shown for both cases. The value of $\hat{o}_{1}$ for the best-case is 0.038 and for the worst case is $\mathbf{0 . 3 8 4}$. 